\title{
Enhanced nucleotide excision repair capacity in lung cancer cells by preconditioning with DNA-damaging agents
}

\author{
Ji Ye Choi ${ }^{1}$, Jeong-Min Park ${ }^{1}$, Joo Mi Yi' ${ }^{2}$, Sun-Hee Leem ${ }^{1}$, Tae-Hong Kang ${ }^{1}$ \\ ${ }^{1}$ Department of Biological Science, Dong-A University, Busan, Korea \\ ${ }^{2}$ Research Center, Dongnam Institute of Radiological \& Medical Sciences, Busan, Korea \\ Correspondence to: \\ Tae-Hong Kang, e-mail: thkang@dau.ac.kr
}

Keywords: nucleotide excision repair, XPA, SIRT1, chemoresistance

Received: January 28, $2015 \quad$ Accepted: June 20, $2015 \quad$ Published: July 20, 2015

\section{ABSTRACT}

The capacity of tumor cells for nucleotide excision repair (NER) is a major determinant of the efficacy of and resistance to DNA-damaging chemotherapeutics, such as cisplatin. Here, we demonstrate that using lesion-specific monoclonal antibodies, NER capacity is enhanced in human lung cancer cells after preconditioning with DNA-damaging agents. Preconditioning of cells with a nonlethal dose of UV radiation facilitated the kinetics of subsequent cisplatin repair and vice versa. Dualincision assay confirmed that the enhanced NER capacity was sustained for 2 days. Checkpoint activation by ATR kinase and expression of NER factors were not altered significantly by the preconditioning, whereas association of XPA, the rate-limiting factor in NER, with chromatin was accelerated. In preconditioned cells, SIRT1 expression was increased, and this resulted in a decrease in acetylated XPA. Inhibition of SIRT1 abrogated the preconditioning-induced predominant XPA binding to DNA lesions. Taking these data together, we conclude that upregulated NER capacity in preconditioned lung cancer cells is caused partly by an increased level of SIRT1, which modulates XPA sensitivity to DNA damage. This study provides some insights into the molecular mechanism of chemoresistance through acquisition of enhanced DNA repair capacity in cancer cells.

\section{INTRODUCTION}

Effective treatment of cancers usually requires the use of genotoxic chemotherapy. In most cases, multiple drugs are used, as resistance to single agents occurs almost universally. However, this causes many side effects for patients and thus, elucidation of mechanisms that confer chemoresistance has been a major goal of cancer biologists for decades. Cisplatin is one of the most commonly prescribed chemotherapeutic agents and produces platinum-DNA adducts, including intra- and inter-strand adducts and DNA-protein crosslinks $[1,2]$. In humans, these lesions are removed primarily by nucleotide excision repair (NER) and hence, the status of NER is a critical indicator of the success of chemotherapy with cisplatin [3-5]. The importance of NER is highlighted by the finding that defects in this pathway result in hypersensitivity to cisplatin, and that restoration of NER activity reduces sensitivity to more normal levels $[6,7]$. In spite of the critical role of NER in cisplatin resistance in cancer cells, however, the change in NER kinetics upon sequential cisplatin treatment or upon cisplatin treatment followed by treatment with another type of NER-dependent DNAdamaging agent, such as UV radiation, has not been precisely investigated due to the limitation of analytical tools such as cisplatin-DNA adduct-specific monoclonal antibody.

NER is a complex process carried out by seven xeroderma pigmentosum (XP) proteins (XPA to XPG) and approximately two dozen non-XP proteins [8]. Importantly, upregulation of only a few rate-limiting components of the NER system is necessary to increase a cell's capacity for NER. One such important rate-limiting factor is XPA, which has been found to be overexpressed in cisplatin-resistant cancers [9, 10]. In ovarian cancer, XPA was shown to be expressed at a higher level in tumors of patients resistant to cisplatin treatment [11]. Low levels of XPA are expressed in testicular cancer, which is generally very responsive to cisplatin, providing further correlative evidence for the importance of NER 
in cisplatin resistance $[12,13]$. The rate-limiting effect of XPA in NER has been demonstrated in normal human fibroblasts (NHFs) as well. When XPA was downregulated to $60 \%, 10 \%$, and $4 \%$ of its original value in the NHF-1 cell line in a controlled manner by titrating the amount of $X P A$ siRNA, the rates of NER of both the 6-4 photoproduct (6-4PP) and the cyclobutane pyrimidine dimer (CPD), the two major types of DNA lesion caused by UV irradiation, were proportionally reduced [14]. Although the rate of repair of CPDs was linearly correlated with the level of XPA, the rate of 6-4PP repair exhibited a parabolic relationship with the XPA level, which is consistent with the well-established fact that the 6-4PP is repaired at a 5-10-fold faster rate than the CPD [15].

The steady-state level of XPA is mainly controlled by the circadian clock $[16,17]$, HERC2 [18, 19] and SIRT1 [20, 21]. The transcriptional activity of the circadian clock induces a daily rhythm of XPA gene expression, whereas HERC2 functions as an E3 ubiquitin ligase for XPA degradation in a proteasome-dependent fashion. The half-life of XPA protein is approximately $4 \mathrm{~h}$ in the absence of DNA damage, but much longer in the presence of DNA damage [19]. In response to DNA damage, XPA is phosphorylated by ATR kinase, which stabilizes XPA by preventing its association with HERC2 [18]. Thus, ATR activity in response to DNA damage can be utilized to a certain extent as a surrogate marker for NER activity. SIRT1, a $\mathrm{NAD}^{+}$-dependent histone deacetylase, also plays a critical role in the NER pathway. A recent study revealed that SIRT1 can deacetylate XPA and that this is required for interaction with replication protein A (RPA) and optimal NER activity [20].

Because of the significance of the NER capacity in a mechanism of chemoresistance in cancer cells, we decided to investigate the change in NER capacity after preconditioning of cells with a nonlethal dose of a DNAdamaging agent, which generates cells that mimic resistant cells after chemotherapy. For NER kinetic analysis we employed lesion-specific monoclonal antibodies to detect UV-induced CPD or 6-4PP and cisplatininduced platinum-GpG adduct [22]. We found that the preconditioning renders cancer cells more resistant to a subsequent lethal dose of DNA-damaging agent by modulating the sensitivity of XPA association with DNA lesions hence, enhancing NER activity and conferring chemoresistance on cancer cells.

\section{RESULTS}

In order to obtain insight into the effect of DNA repair capacity on the mechanism of chemoresistance, we investigated changes in NER activity after treatment of cells with nonlethal doses of DNA-damaging agents. We used two monoclonal antibodies to specifically detect UVinduced CPDs and Pt-GpG adducts, lesions that are the exclusive substrates of NER $[3,4]$. To exclude the effect of cell cycle on DNA repair activity, human non-small cell lung carcinoma A549 and large cell lung carcinoma H460 cells were grown to confluence and kept for additional four days to completely block cell proliferation (Figure 1A-1C) before treatment with DNA damaging agents. Several UV doses were applied to measure the repair activity and cell viability. The amount of CPD lesions on genomic DNA was analyzed by immunoslot blotting (Figure 1D), and cell viability after $24 \mathrm{~h}$ of UV exposure was assessed by a fluorescence-based cell viability assay (Figure 1E). Upon irradiation with $5 \mathrm{~J} / \mathrm{m}^{2}$ $\mathrm{UV}$, there was no significant decrease of cell number and $24 \mathrm{~h}$ was sufficient for complete repair of CPDs. However, irradiation of cells with more than $5 \mathrm{~J} / \mathrm{m}^{2}$, including 10 or $20 \mathrm{~J} / \mathrm{m}^{2}$, resulted in substantial decrease in cell number, and CPDs still remained on genomic DNA at $24 \mathrm{~h}$ after UV irradiation. Based on these results, we chose $5 \mathrm{~J} / \mathrm{m}^{2}$ of UV dose as a repairable and nonlethal condition for activation of DNA damage response in lung cancer cells, which behaved like recurrent cancer or chemoresistant cells after primary chemotherapy with DNA-damaging agents. We termed this condition as "preconditioning" (PreC) and examined whether it affected subsequent DNA repair activity evoked by cisplatin treatment at a lethal concentration of $10 \mu \mathrm{M}$. As shown in Figure $1 \mathrm{~F}$ and $1 \mathrm{G}$, UV-PreC facilitated repair of subsequent Pt$\mathrm{GpG}$ adduct compared to the nonpreconditioned control (nonPreC). In the inverse experiment, we preconditioned cells with a nonlethal concentration of cisplatin $(5 \mu \mathrm{M})$ and then investigated repair of the UV-induced CPDs. As expected, repair of CPDs caused by $10 \mathrm{~J} / \mathrm{m}^{2} \mathrm{UV}$ required more time than those induced by $5 \mathrm{~J} / \mathrm{m}^{2} \mathrm{UV}$ (Figure 2A, lanes 1 and 2). This pattern was also observed when cells were preconditioned with cisplatin (Figure 2A, lanes 4 and 5). However, the kinetics of CPD repair after the same dose of UV were much faster when cells had been preconditioned with cisplatin (Figure 2B and 2C), which is similar to the effect of UV-PreC shown in Figure 1. Next, we measured Pt-GpG removal rate following Pt-PreC. As shown in Figure 3A, there was no remaining Pt-GpG adduct after $48 \mathrm{~h}$ of Pt-PreC with $5 \mu \mathrm{M}$ of cisplatin. Meanwhile the repair kinetics upon following cisplatin treatment was much faster in Pt-PreC than nonPreC cells (Figure 3B). These results suggest that NER activity may be upregulated by PreC with a nonlethal dose of DNA-damaging agent. To test this hypothesis we measured the cell's NER capacity at specific times from $12 \mathrm{~h}$ to $96 \mathrm{~h}$ after PreC. To this end, we used an in vitro dual-incision assay, for which we prepared DNA substrate containing UV-induced 6-4PP, which is a better substrate than CPD, and cell lysate prepared at various time points after PreC. Figure 4 shows that the lysate of nonPreC cells had no time-dependent effect on dual-incision activity, whereas the lysate of Pt-PreC cells showed changes in NER capacity depending on the duration of time after PreC. NER activity started to increase $12 \mathrm{~h}$ after PreC and 
peaked at $48 \mathrm{~h}$, at which time the lesions were completely repaired. However the enhancement of NER capacity by PreC was no longer detected $72 \mathrm{~h}$ after PreC (Figure 4).

To decipher the mechanism underlying enhancement of NER capacity by PreC, we first analyzed the levels of core NER factors XPA through XPG at $24 \mathrm{~h}$ after PreC and compared this with levels in nonpreconditioned controls because some previous reports demonstrated increase of core NER factors including XPA and XPF during adaptive response. As shown in Figure 5, however, there was no significant change in the expression of NER factors regardless of PreC. Next, we analyzed ATR kinase activity indirectly by monitoring the level of phosphorylation of its substrate proteins p53 and CHK1. ATR is known to augment NER activity by phosphorylating and, thus stabilizing, XPA in response to DNA damage [18]. The result indicates that UV-PreC had no effect on ATR activity as no significant alteration in phosphorylation of p53 or CHK1 was detected after PreC (Figure 5). In addition, similar phosphorylation profiles were obtained from nonPreC and UV-PreC cells, which implies that ATR activity had not been altered by PreC.

XPA is the key rate-limiting factor for NER [11, 14]. However, given that PreC had no effect on XPA protein level or ATR activity, we next examined the effect of PreC on XPA mobility to damaged DNA using local UV irradiation. XPA foci at locally-exposed sites strongly coincided with 6-4PP lesions (Figure 6A). As similar as shown in immunoslot blot data in previous figures, Pt-PreC accelerated the 6-4PP removal than nonPreC control, as demonstrated by more rapid disappearance of 6-4PP signal (Figure 6A). For a quantitative analysis we

A

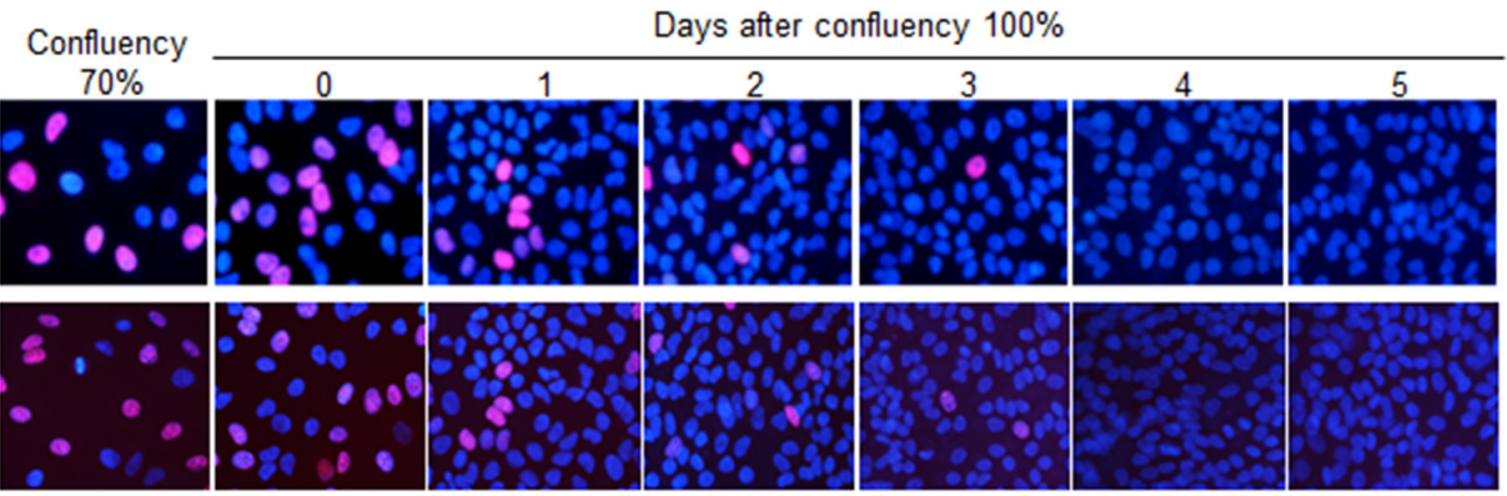

A549

EdU/Hoechst

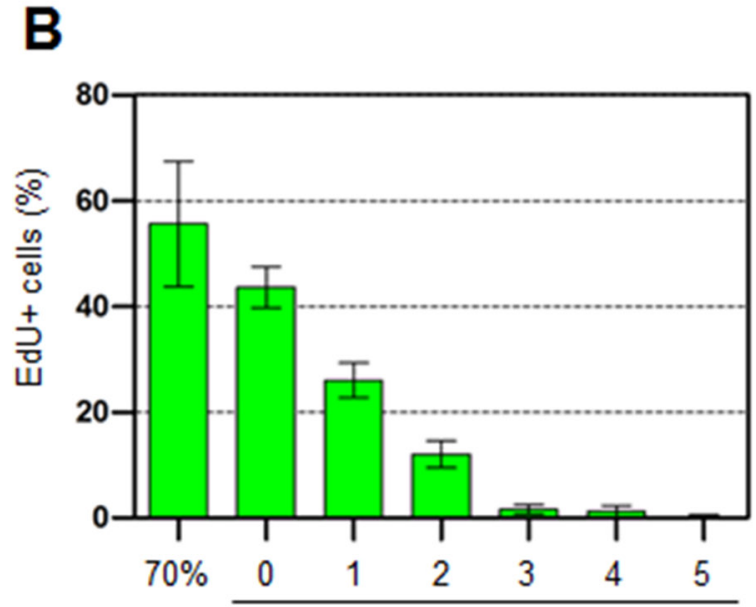

Days after confluency $100 \%$

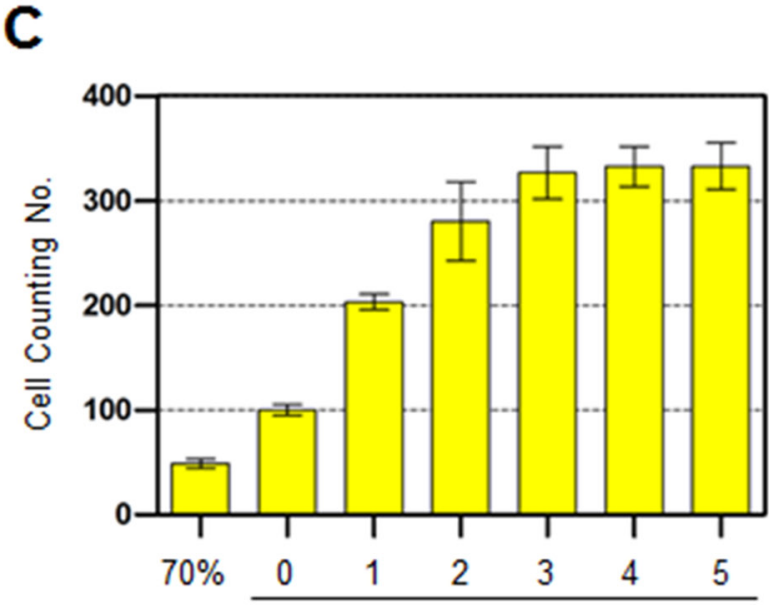

Days after confluency $100 \%$

Figure 1: Preconditioning of cells with UV irradiation facilitates subsequent repair of cisplatin-induced damage. A. A549 and H460 lung carcinoma cells were grown to the indicated density $70 \%$ or $100 \%$. The $100 \%$ confluency is designated at the time when there is no space among the cells. EdU was added $2 \mathrm{~h}$ before fixation at the indicated culture density and days after $100 \%$ confluent. B. EdU-positive A549 cell numbers were counted among 1000 cells. C. The number of A549 cells in at day 0 of $100 \%$ confluent was designated as 100 control. The cell numbers from the other samples were plotted as relative values compared to control. The bars and error bars represent the mean $\pm \operatorname{s.d}(n=3)$. (Continued) 

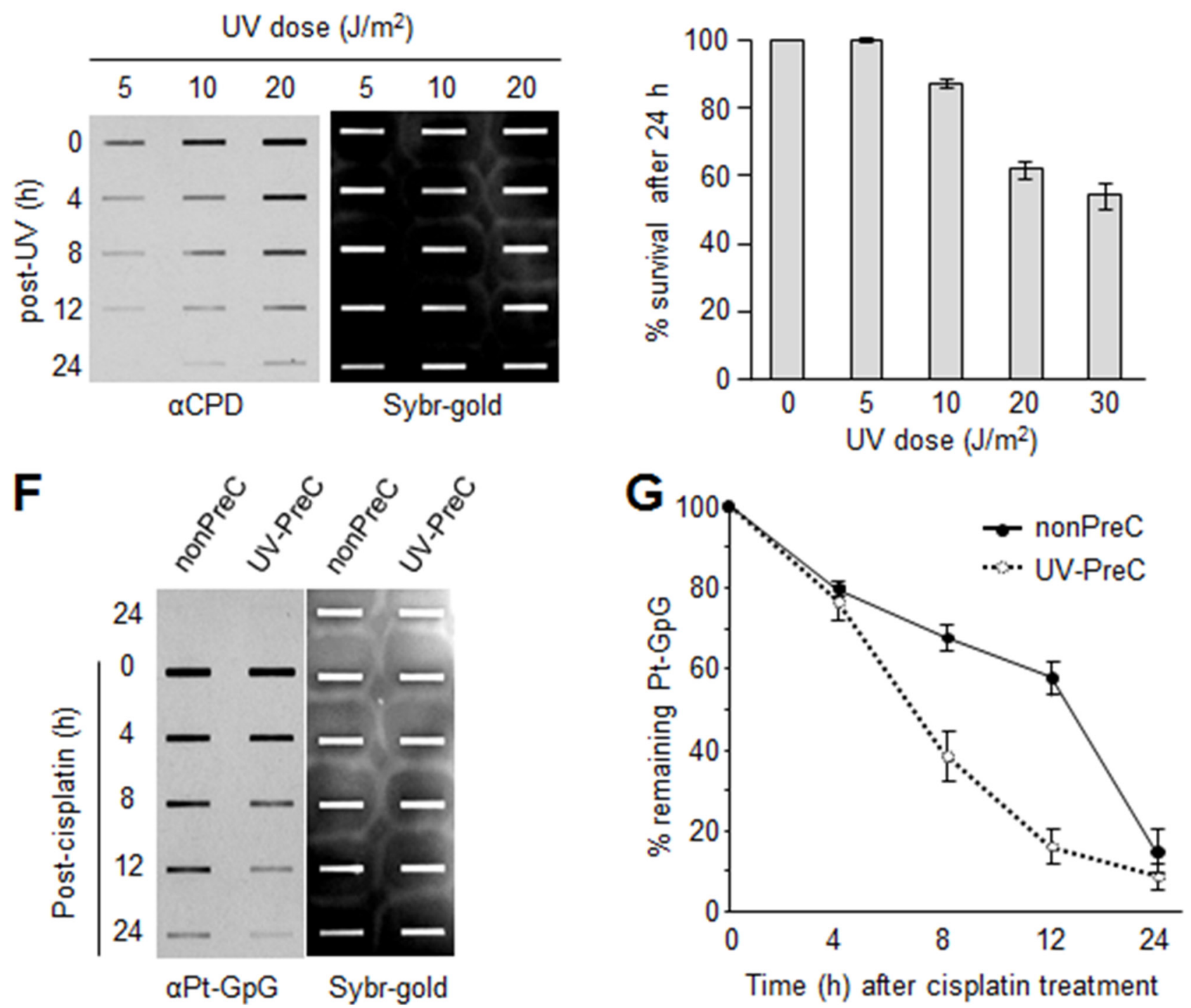

Figure 1: Preconditioning cells with UV irradiation facilitates subsequent repair of cisplatin-induced damage. (Continued) D. A549 cells irradiated with the indicated UV doses were allowed to carry out repair for the indicated times, followed by isolation of genomic DNA and immunoslot blotting analysis to detect residual cyclobutane pyrimidine dimers (CPDs). After immunoslot blotting, the membrane was counterstained with SYBR-Gold for a loading control of genomic DNA. E. Cell viability after UV irradiation was assessed by fluorescence-based cell viability assay. Constitutive protease activity within live cells was measured using a fluorogenic and cell permeable peptide substrate using CellTiter-Fluor Cell Viability Assay kit. The fluorescent signal obtained from mock-treated cells was designated as 100 and the relative values obtained from UV-exposed cells were plotted. The bars and error bars represent the mean \pm s.d $(n=3)$. F. Removal rates of platinum-GpG (Pt-GpG) from nonpreconditioned (nonPreC) or UV-preconditioned (UV-PreC) cells. Cells were either mock-treated (nonPreC) or $5 \mathrm{~J} / \mathrm{m}^{2}$ of UV-treated (UV-PreC) and kept for $24 \mathrm{~h}$ and followed by $10 \mu \mathrm{M}$ of cisplatin treatment for $2 \mathrm{~h}$ and then culture medium was changed to wash out residual cisplatin in the medium. Recovery times were allowed for the indicated times and genomic DNAs obtained from each time point were assessed by immunoslot blotting using Pt-GpG-specific monoclonal antibody. G. The quantitative analysis for (F). The bars and error bars represent the mean \pm s.d from three independent experiments.

counted the number of XPA foci-positive cells and found no difference between nonPreC and Pt-PreC within 30 $\mathrm{min}$ of recovery time (Figure 6B). However, at $60 \mathrm{~min}$ after UV exposure approximately 3 times less XPA focipositive cells were detected in Pt-PreC, indicating that the Pt-PreC may modulate the efficient XPA recruitment on DNA lesions followed by a robust repair and possibly conferring resistance to toxic DNA damage. Because SIRT1, a histone deacetylase, has been implicated recently in the NER pathway by virtue of deacetylating XPA and thus enhancing NER activity, we measured the level of SIRT1. Interestingly, UV-PreC cells showed 
$\mathbf{A}$

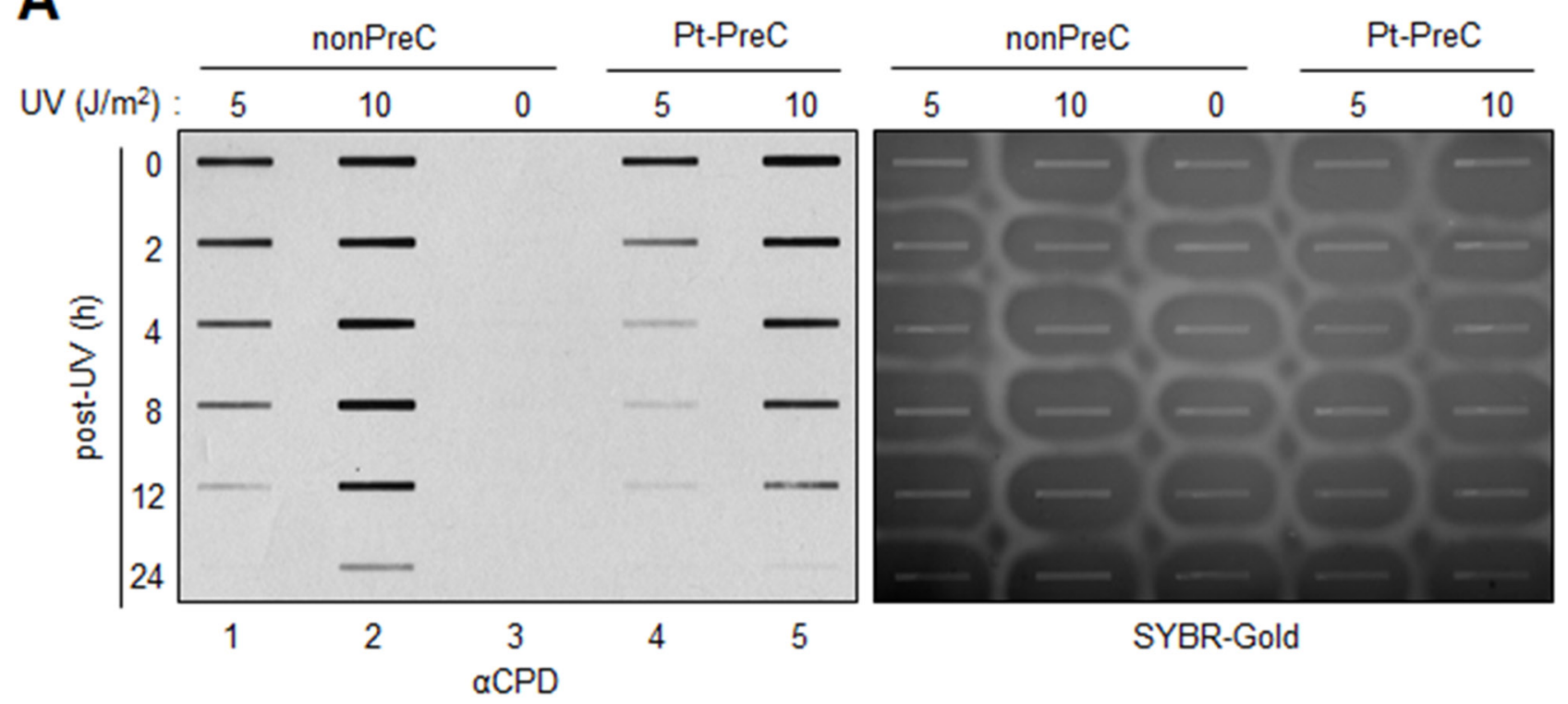

B
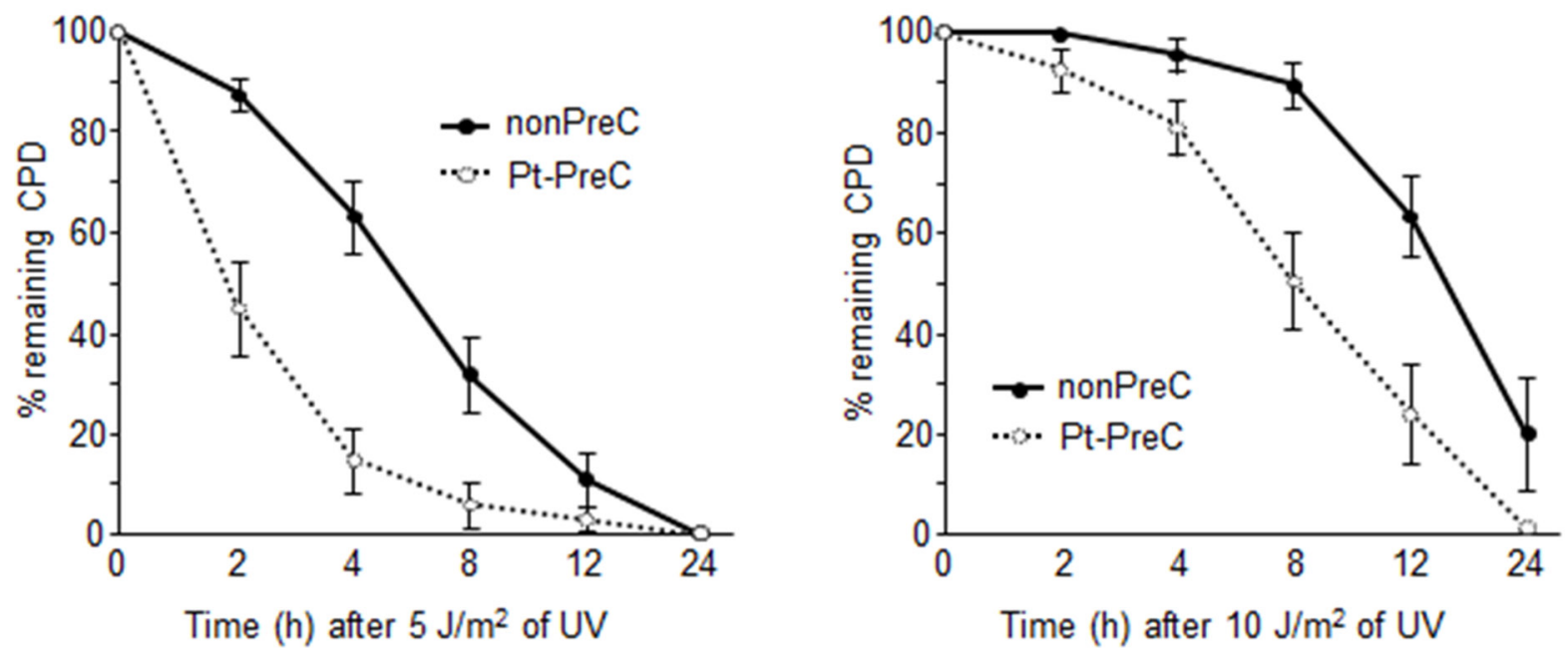

Figure 2: Enhanced UV-induced CPD repair activity following Pt-PreC. A. The residual CPDs in genomic DNA of nonpreconditioned cells (nonPreC) or cells preconditioned with $5 \mu \mathrm{M}$ of cisplatin (Pt-PreC) were assessed by immunoslot blotting. After immunoslot blotting the membrane was counterstained with SYBR-Gold for a loading control of genomic DNA. B. $5 \mathrm{~J} / \mathrm{m}^{2}$ or C. $10 \mathrm{~J} / \mathrm{m}^{2}$ of UV- induced CPD repair kinetics were measured from cells conditioned with nonPreC or Pt-PreC.

increased levels of SIRT1 compared to nonPreC control (Figure 7A). To verify the acetylation status of XPA we immunoprecipitated XPA and determined the acetylation level with anti-acetyl-lysine antibody. The result indeed indicated a decrease in acetylation level of XPA with UV-PreC, which is immediately reversed by treatment of SIRT1 inhibitor EX527 (Figure 7B). To confirm the role of SIRT1 in the PreC effect, we pretreated cells with the specific SIRT1 inhibitor EX-527 [23] before treatment of cisplatin following the UV-PreC and investigated XPA loading to chromatin. UV-PreC-induced enhancement of XPA chromatin loading was reduced in the presence of EX527, implying that the SIRT1 regulated XPA acetyl status may contribute XPA sensitivity to DNA lesions. The PreC-induced repair capacity was also compromised by SIRT1 inhibition (Figure 7C), which implies that upregulation of SIRT1 is the major mechanism in PreCinduced NER potentiation.

\section{DISCUSSION}

An ultimate goal in cancer therapy is to devise individually tailored treatment plans that target growthpromoting pathways and circumvent drug resistance. In general, tumor cells acquire resistance by manipulating biochemical mechanisms that reduce pharmacokinetics 


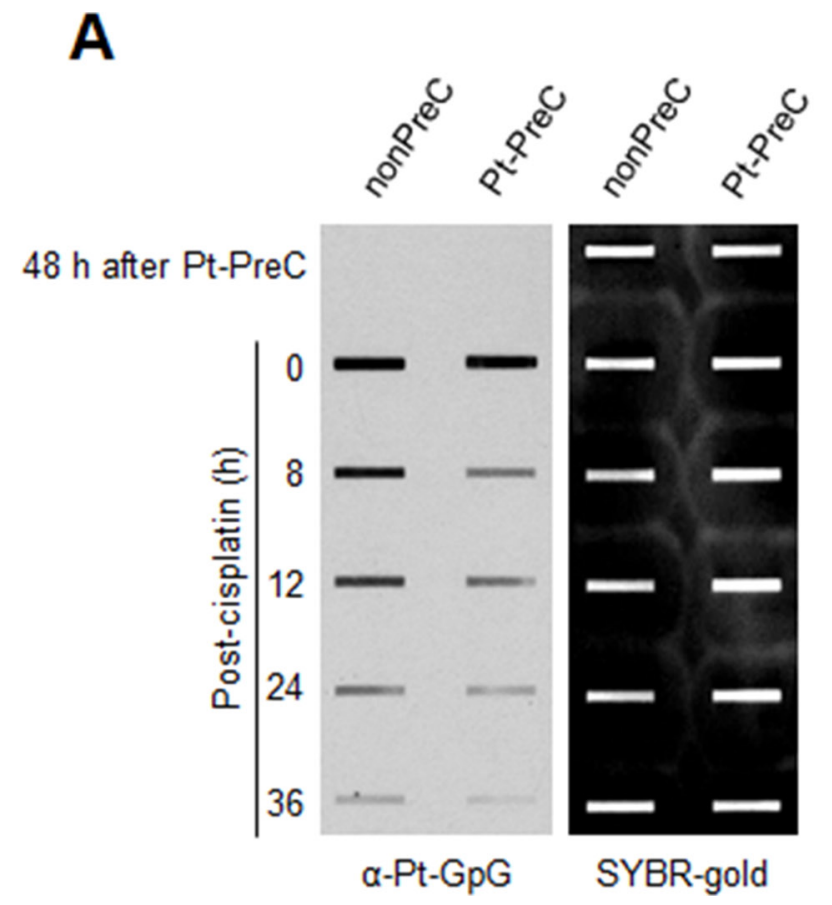

B

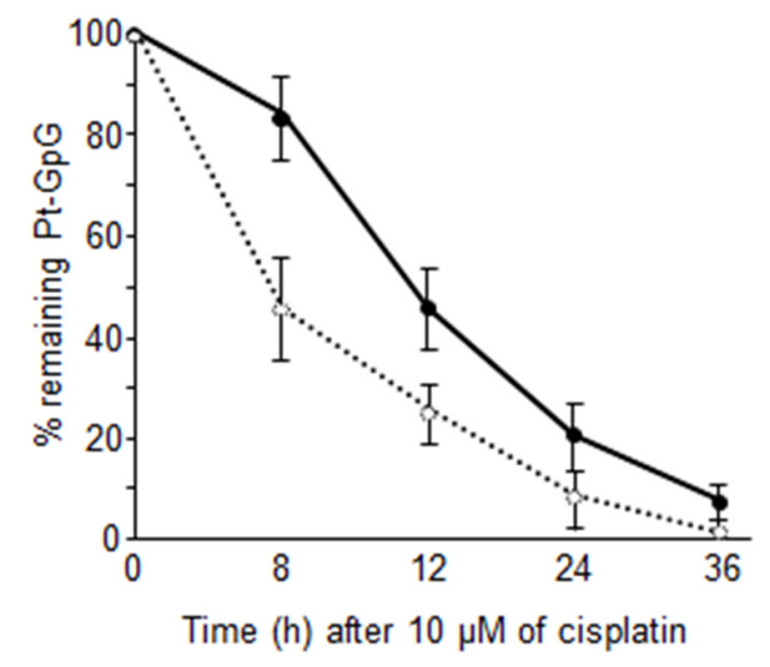

Figure 3: Enhanced Pt-GpG adduct removal by Pt-PreC. A. Cells preconditioned with $5 \mu \mathrm{M}$ of cisplatin or mock were treated with $10 \mu \mathrm{M}$ of cisplatin, and Pt-GpG adduct removal rate was measured using immunoslot blotting with Pt-GpG adduct-specific monoclonal antibody. B. The quantitative analysis for (A) The bars and error bars represent the mean \pm s.d from three independent experiments.

or by acquiring additional alterations in DNA damage response pathways. Hence, an understanding of these processes is important for predicting treatment response and for the development of novel treatment strategies for chemoresistance. Most chemotherapeutics rely for their anticancer activity on induction of a DNA damage response to promote the apoptotic pathway [24, 25]. However, DNA repair pathways counteract this effect by repairing damaged DNA and restoring it to normal status. Cisplatin, carboplatin, and oxaliplatin are platinum-based drugs for treatment of many types of cancers, including head and neck, testicular, ovarian, cervical, lung, colorectal, and relapsed lymphoma [3-5, 7]. The cytotoxicity of platinating agents is thought to be due to the platinum intrastrand crosslink that forms on DNA, such as Pt-GpG adduct $[3,4]$. Resistance can be caused by a number of cellular adaptations, including reduced uptake, inactivation by intracellular antioxidants, and increased DNA repair capacity $[3,7]$. In this report, we identified a plausible mechanism of chemoresistance by which NER capacity is enhanced by preconditioning of cells with a nonlethal dose of DNA-damaging agents (PreC). By virtue of lesionspecific antibodies we were able to precisely measure the repair kinetics caused by specific DNA damage. Pt-GpG adduct removal following UV-PreC, UV-CDP or UV-6-4PP removal following Pt-PreC and $\mathrm{Pt}-\mathrm{GpG}$ removal following Pt-PreC were enhanced compared to nonPreC control, which led us the general conclusion that PreC makes cells more resistant to subsequent toxic DNA damage at least in lung carcinoma A549 and H460 cells. Some earlier studies had already shown the adaptive response evoked by preconditioning of which are mostly driven by increase of core NER factor expression. For instance, preconditioning cells with a low dose of ionizing radiation (IR) enhances UV-induced NER [26]. In response to IR, transcription of NER genes, such as XPC and DDB2 (XPE), was upregulated due to the stabilization of $\mathrm{p} 53$, which induces transcription of the genes. However, in this study we did not detect such an increase in any core NER factors; nor did we detect p53 activation. Instead we found a novel pathway for an adaptive response mediated by upregulation of SIRT1. Because the expression level of XPA, the rate limiting factor in NER was not changed by PreC we assumed that posttranslational modification of XPA occurred during PreC, but that was not mediated by ATR because ATR activity was not altered by PreC. Importantly, we found that increased expression of SIRT1 upon PreC compared to nonPreC control. Reportedly SIRT1 contributed to the decrease of acetylated XPA or increase of deacetylated XPA [20] that showed preferential binding to the DNA damage, required for the enhanced NER capacity induced by PreC. Antagonizing SIRT1 activity using inhibitors such as EX527, sirtinol, and the tenovins has been demonstrated to induce p53-mediated apoptosis [27-29], suggesting that SIRT1 inhibition may be beneficial for treating certain types of cancer. Here we support the SIRT1's role in chemoresistance in some types of lung cancer cells and demonstrate that using SIRT1 inhibitor to treat a certain 


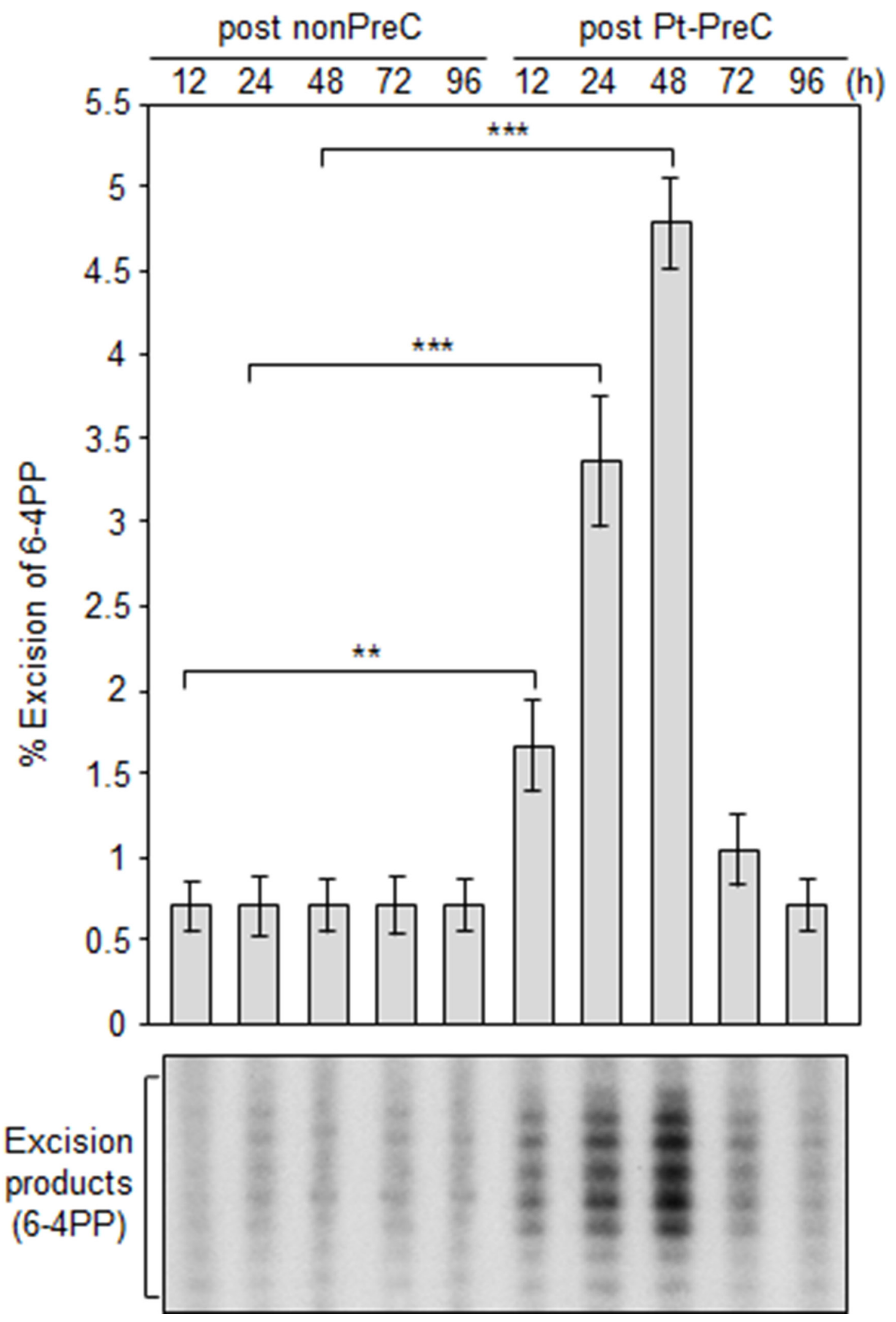

Figure 4: Pt-PreC enhances NER capacity for 6-4 photoproduct (6-4PP) removal. Dual-incision NER activity assay was performed using isotope-labeled and 6-4PP-containing linear substrate DNA and cell lysates obtained from nonPreC and Pt-PreC cells at the indicated times after PreC. Amount of excision product was used as a measure of the NER capacity of the lysate. Results are presented as mean $\pm \mathrm{SD}$ from three independent experiments. Differences were considered significant at the values of $P<0.01(* *)$ and $P<0.001(* * *)$. 


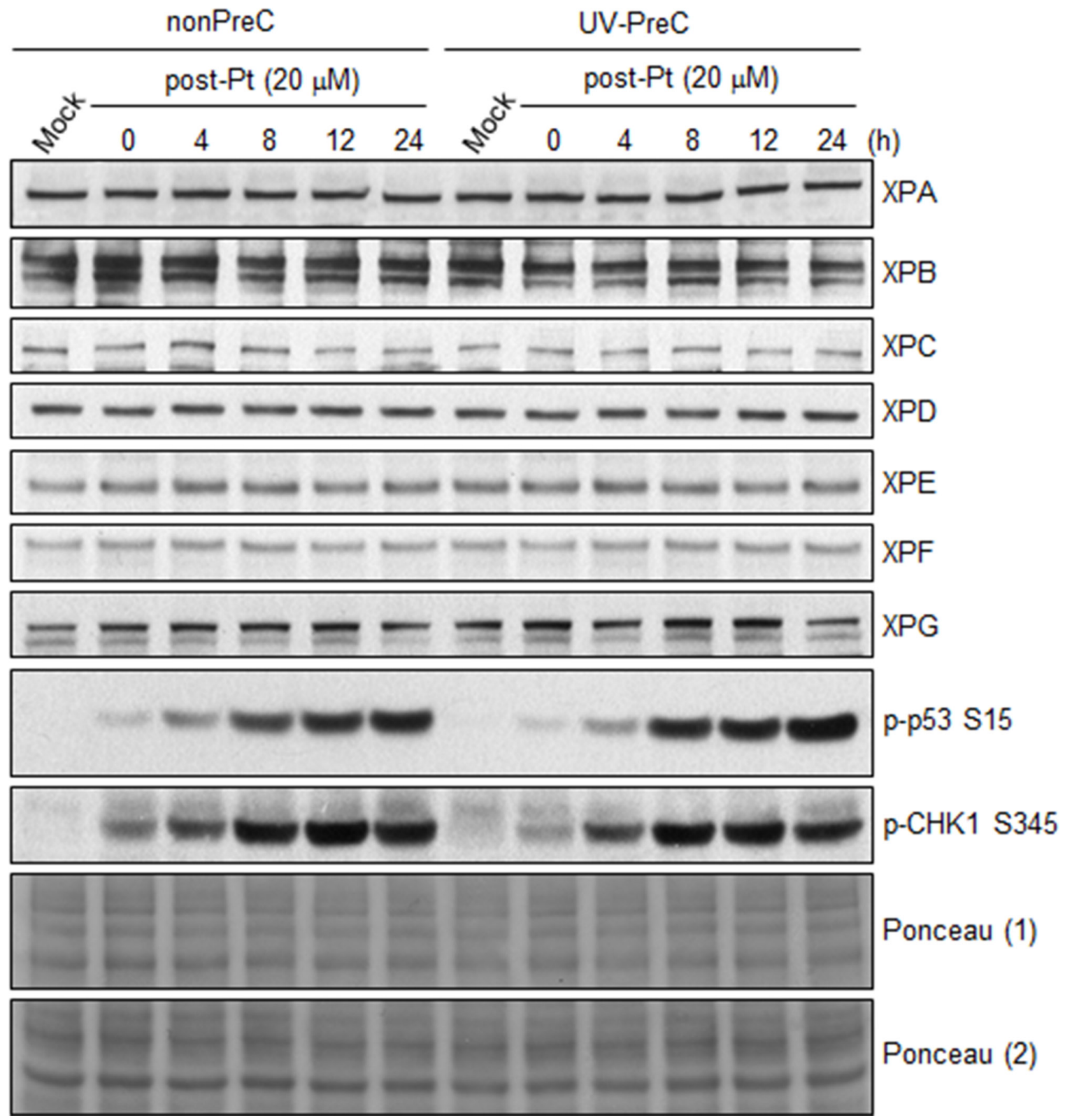

Figure 5: PreC does not alter the protein expression of core NER factors nor ATR activity. $24 \mathrm{~h}$ later of UV-PreC with $5 \mathrm{~J} / \mathrm{m}^{2}$, cells were treated with $20 \mu \mathrm{M}$ of cisplatin for $2 \mathrm{~h}$ and then cells were allowed to recover for the indicated times. Protein levels of core NER factors (XPA-XPG) and ATR substrate proteins (p-p53 and p-CHK1) were assessed by immunoblotting with the indicated antibodies. Ponceau stained blots from two different gels were used to indicate equal loading of the samples.

type of lung cancer showing acquired NER capacity would be beneficial for cancer treatment.

\section{MATERIALS AND METHODS}

\section{Cell culture and cell viability assay}

A549 and H460 cells (American Type Culture Collection, Manassas, VA, USA) were cultured in Dulbecco's modified Eagle medium supplemented with $10 \%$ fetal bovine serum and 1\% penicillin-streptomycin. $100 \%$ confluent cells were kept for additional four days to completely block cell proliferation and then exposed to the UV light using a germicidal lamp (for immnoslot blotting) or UV crosslinker (for immunofluorescence) emitting primarily UV-C light. A UV-C sensor (UV Products, Upland, CA, USA) was used to calibrate the fluence rate of the incident light. For immunofluorescence staining, cells were grown on a glass coverslip coated with poly-Dlysine and laminin (BD Biosciences, San Jose, CA, USA). For assessment of cell viability, CellTiter-Fluor Cell Viability Assay kit (Promega, Madison, WI, USA) was used as indicated in manufacturer's protocol. Fluorescence plate reader (BioRad, Hercules, CA, USA) was used to 
A

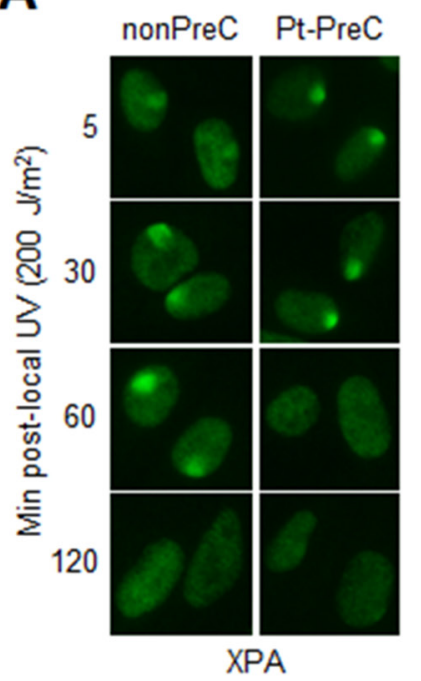

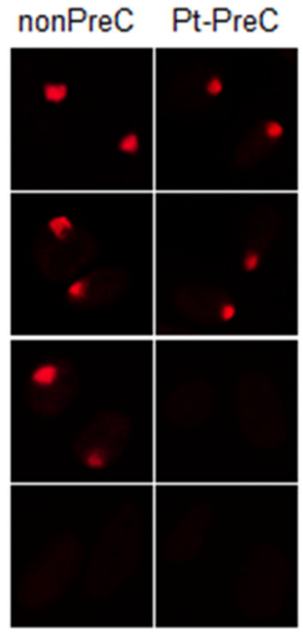

6-4PP

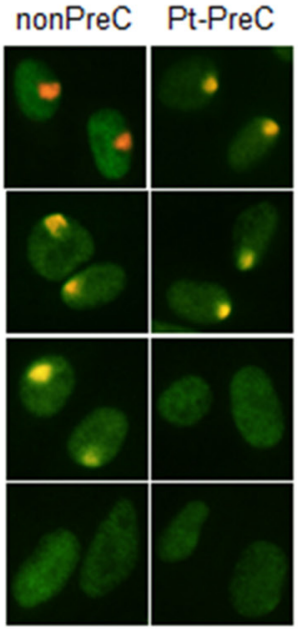

merge

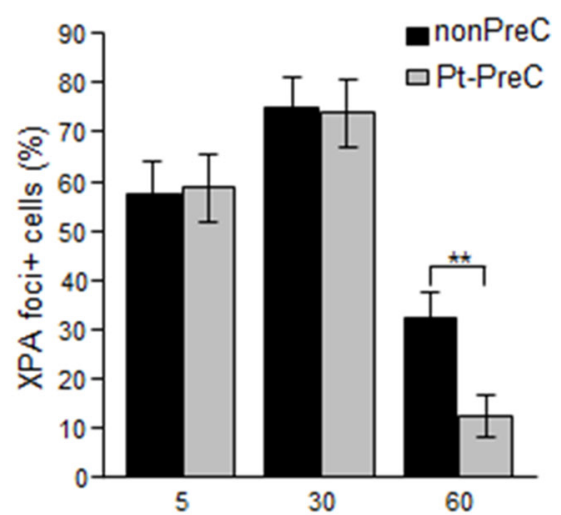

Min post-local UV $\left(200 \mathrm{~J} / \mathrm{m}^{2}\right)$

Figure 6: PreC accelerates XPA binding to DNA lesions. A. 6-4PP removal kinetics and lesion-specific XPA binding from cells preconditioned with nonPreC control or Pt-PreC were monitored after $200 \mathrm{~J} / \mathrm{m}^{2}$ of local UV irradiation using isopore filter with $5 \mu \mathrm{m}$ in diameter. B. The number of XPA foci-positive cells was calculated among 1000 cells counted. Results are presented as mean \pm SD from three independent experiments. Differences were considered significant at the value of $P<0.01(* *)$.

measure a constitutive protease activity within live cells using a fluorogenic and cell permeable peptide substrate.

\section{Immunoslot blotting}

Genomic DNA was obtained using a QIAamp DNA Mini Kit (Qiagen, Hilden, Germany), and $100 \mu \mathrm{g}$ (for CPD) or $500 \mu \mathrm{g}$ (for 6-4PP and platinum [Pt]-GpG adduct) DNA was vacuum-transferred to a nitrocellulose membrane using a BioDot SF Microfiltration apparatus (BioRad). DNA was crosslinked to the membrane by incubation at $80^{\circ} \mathrm{C}$ for $2 \mathrm{~h}$ under vacuum. Monoclonal antibodies that recognize CPD (Kamiya, Seattle, WA, USA), 6-4PP (Cosmo Bio, Tokyo, Japan) and Pt-GpG (Oncolyze, Essen, Germany) were used to detect the amounts of remaining lesions in the genomic DNA. After the immunoslot blot assay, the total DNA amounts loaded onto the membrane were visualized with SYBR-gold staining, and these values were used for normalization.

\section{Dual-incision NER activity assay}

Assay of NER activity in the cell lysate toward 6-4PP-containing DNA substrate was carried out as reported previously [30]. Briefly, 10 fmol of 140-bp duplex with a 6-4PP in the center and ${ }^{32} \mathrm{P}$-label at the 5th phosphodiester bond 5' to the site of the lesion was incubated with $70 \mu \mathrm{g}$ of lysate in $25 \mu \mathrm{L}$ of excision buffer at $30^{\circ} \mathrm{C}$ for $1 \mathrm{~h}$. The amount of excision product was used as a measure of NER capacity in the lysate. The 6-4PP-containing linear duplex substrate DNA and NER-competent cell lysate were prepared as described previously [16].

\section{Immunoblotting and immunoprecipitation}

Whole-cell lysate prepared as described [31] was used to determine the levels of proteins. Antibodies used in this study include those against XPA (Kamiya), XPBXPD (Santa Cruz Biotechnology, Santa Cruz, CA, USA), XPE, p-p53, p-CHK1, GAPDH, SIRT1, acetyl-lysine (Cell Signaling Technology), XPF, and XPG (both Abcam, Cambridge, UK). For immunoprecipitation of XPA, $1 \mathrm{mg}$ of whole-cell lysate was incubated with $1 \mu \mathrm{g}$ of antiXPA conjugated to Protein A/G-agarose beads (Sigma, St. Louis, MO, USA) for $12 \mathrm{~h}$ at $4{ }^{\circ} \mathrm{C}$ with rotation. After washing with lysis buffer, proteins were eluted from the beads by boiling in SDS sample buffer and resolved on $10 \%$ SDS-polyacrylamide gels. For detection of XPA acetylation, anti-acetyl-lysine was employed.

\section{Local UV irradiation and immunofluorescence}

Cells preconditioned with cisplatin were irradiated with UV-C at a dose of $200 \mathrm{~J} / \mathrm{m}^{2}$ through an isopore polycarbonate filter with pores $5 \mu \mathrm{m}$ in diameter (EMD Millipore). After platinum preconditioning (PtPreC), if necessary, cells were treated with $1 \mu \mathrm{M}$ of specific SIRT1 inhibitor EX-527 (Sigma) for $5 \mathrm{~h}$ before local UV irradiation. After incubation for the recovery times the cells were fixed in $4 \%$ paraformaldehyde for $15 \mathrm{~min}$, followed by conventional immunofluorescence staining procedures. UV-induced lesions were counterlabeled with anti-6-4PP antibody, and XPA foci-positive cells were counted for quantitative analysis. The images were captured using Nikon imaging software NIS-Elements 4.0. 
A

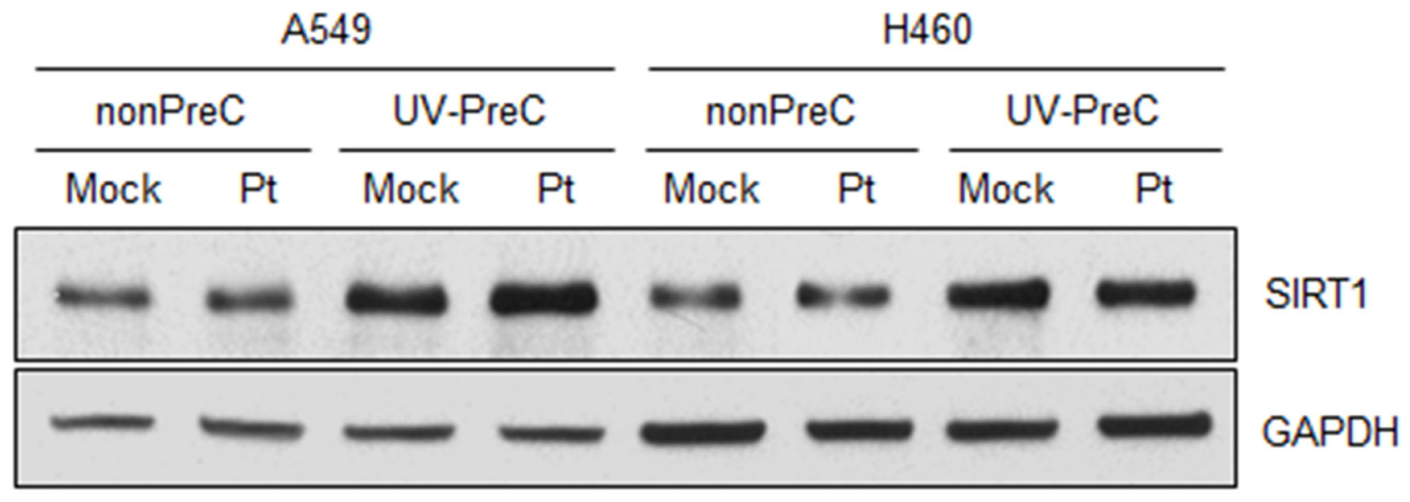
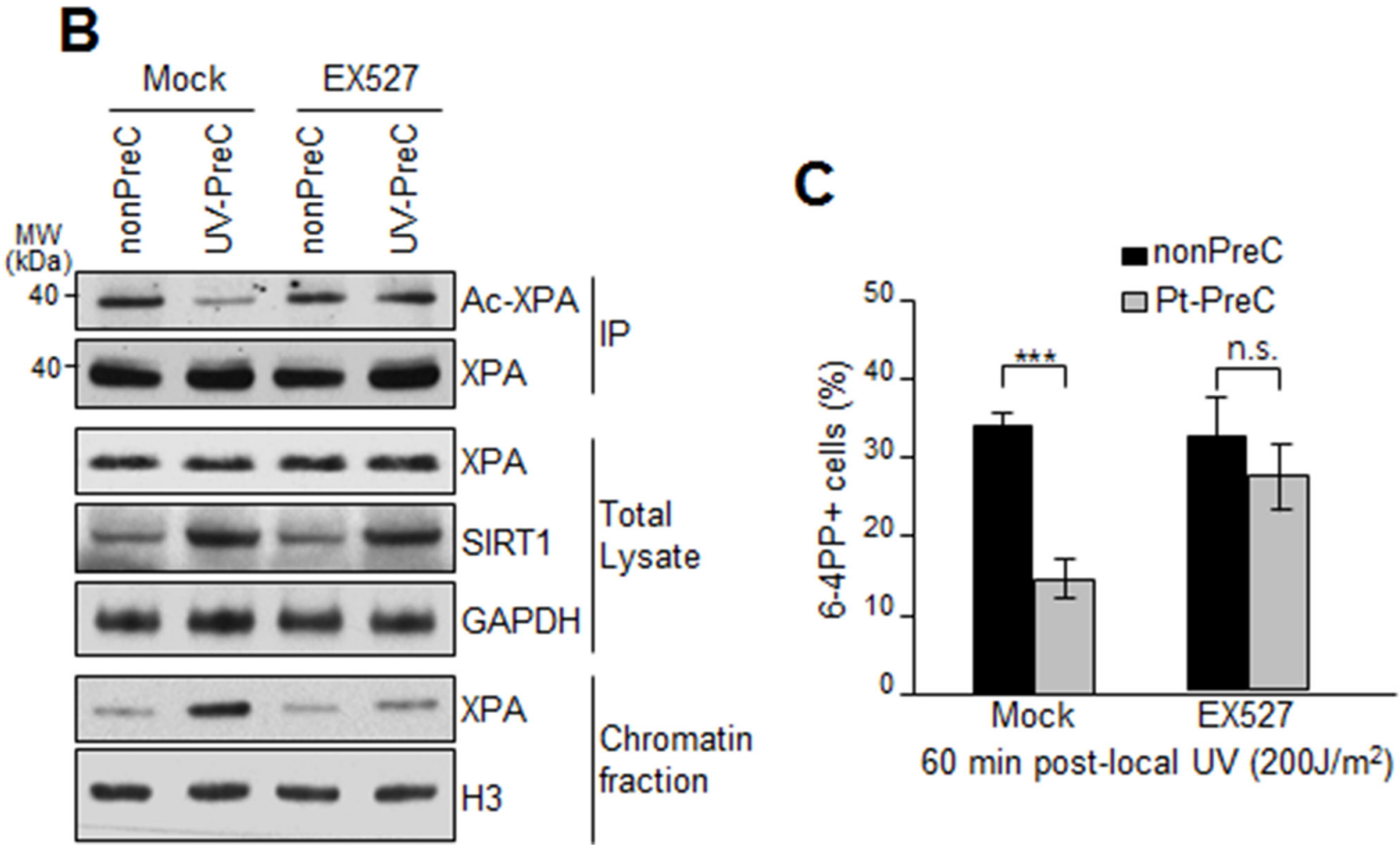

Figure 7: Upregulation of SIRT1 expression during PreC. A. SIRT1 expression from A549 and H460 preconditioned with nonPreC or UV-PreC was assessed by immunoblotting. GAPDH was used as a loading control. B. Acetylation of XPA was assessed by immunoprecipitation of XPA followed by immunoblotting with anti-acetyl-lysine antibody in the presence or absence of EX527, the SIRT1specific inhibitor. PreC cells were pretreated with EX527 for $5 \mathrm{~h}$ before cisplatin treatment. Histone H3 was used to indicate chromatinenriched fraction. C. 6-4PP repair kinetics from nonPreC or Pt-PreC was measured in the presence or absence of EX527. 60 minutes after local UV irradiation the 6-4PP foci-positive cells were counted from randomly selected 1000 cells in each sample. The bars and error bars represent the mean \pm s.d from three independent experiments. Differences were considered significant at the value of $P<0.001(* * *)$.

\section{Statistics}

Data were evaluated using Student's $t$-test, oneway ANOVA with Tukey test, or two-way ANOVA for multiple comparisons as indicated. Results are presented as mean $\pm \mathrm{SD}$ from at least three independent experiments. Differences were considered significant at the values of $P<0.05(*), P<0.01(* *)$, and $P<0.001(* * *)$. Statistical analyses were performed with GraphPad Prism 5.0 software (GraphPad, La Jolla, CA, USA).

\section{ACKNOWLEDGMENTS}

We thank members of DNA repair laboratory in Dong-A University for their technical assistance and helpful discussions. This study was supported by a grant from the National R\&D Program for Cancer Control, Ministry of Health \& Welfare, Republic of Korea (1420070) and National Research Foundation (NRF) of Republic of Korea (NRF-2011-0013804 and NRF-2013R1A2A2A04008115), and Brain Busan 21 Project. 


\section{CONFLICTS OF INTEREST}

The authors declare no conflict of interest.

\section{REFERENCES}

1. Schiller JH, Harrington D, Belani CP, Langer C, Sandler A, Krook J, Zhu J, Johnson DH G. Comparison of four chemotherapy regimens for advanced non-small-cell lung cancer. The New England journal of medicine. 2002; 346:92-98.

2. Jamieson ER, Lippard SJ. Structure, Recognition, and Processing of Cisplatin-DNA Adducts. Chemical reviews. 1999; 99:2467-2498.

3. Wang D, Lippard SJ. Cellular processing of platinum anticancer drugs. Nature reviews Drug discovery. 2005; 4:307-320.

4. Kelland L. The resurgence of platinum-based cancer chemotherapy. Nature reviews Cancer. 2007; 7:573-584.

5. Bowden NA. Nucleotide excision repair: why is it not used to predict response to platinum-based chemotherapy? Cancer letters. 2014; 346:163-171.

6. Olaussen KA, Dunant A, Fouret P, Brambilla E, Andre F, Haddad V, Taranchon E, Filipits M, Pirker R, Popper HH, Stahel R, Sabatier L, Pignon JP, Tursz T, Le Chevalier T, Soria JC, et al. DNA repair by ERCC1 in non-small-cell lung cancer and cisplatin-based adjuvant chemotherapy. The New England journal of medicine. 2006; 355:983-991.

7. Martin LP, Hamilton TC, Schilder RJ. Platinum resistance: the role of DNA repair pathways. Clinical cancer research: an official journal of the American Association for Cancer Research. 2008; 14:1291-1295.

8. Costa RM, Chigancas V, Galhardo Rda S, Carvalho H, Menck CF. The eukaryotic nucleotide excision repair pathway. Biochimie. 2003; 85:1083-1099.

9. Weaver DA, Crawford EL, Warner KA, Elkhairi F, Khuder SA, Willey JC. ABCC5, ERCC2, XPA and XRCC1 transcript abundance levels correlate with cisplatin chemoresistance in non-small cell lung cancer cell lines. Molecular cancer. 2005; 4:18.

10. Honecker F, Mayer F, Stoop H, Oosterhuis JW, Koch S, Bokemeyer C, Looijenga LH. Xeroderma pigmentosum group a protein and chemotherapy resistance in human germ cell tumors. Laboratory investigation; a journal of technical methods and pathology. 2003; 83:1489-1495.

11. Stevens EV, Raffeld M, Espina V, Kristensen GB, Trope CG, Kohn EC, Davidson B. Expression of xeroderma pigmentosum A protein predicts improved outcome in metastatic ovarian carcinoma. Cancer. 2005; 103:2313-2319.

12. Welsh C, Day R, McGurk C, Masters JR, Wood RD, Koberle B. Reduced levels of XPA, ERCC1 and XPF DNA repair proteins in testis tumor cell lines. International journal of cancer Journal international du cancer. 2004; 110:352-361.
13. Mendoza J, Martinez J, Hernandez C, Perez-Montiel D, Castro C, Fabian-Morales E, Santibanez M, GonzalezBarrios R, Diaz-Chavez J, Andonegui MA, Reynoso N, Onate LF, Jimenez MA, Nunez M, Dyer R, Herrera LA. Association between ERCC1 and XPA expression and polymorphisms and the response to cisplatin in testicular germ cell tumours. British journal of cancer. 2013; 109:68-75.

14. Kang TH, Reardon JT, Sancar A. Regulation of nucleotide excision repair activity by transcriptional and post-transcriptional control of the XPA protein. Nucleic acids research. 2011; 39:3176-3187.

15. Mitchell DL. The relative cytotoxicity of (6-4) photoproducts and cyclobutane dimers in mammalian cells. Photochemistry and photobiology. 1988; 48:51-57.

16. Kang TH, Reardon JT, Kemp M, Sancar A. Circadian oscillation of nucleotide excision repair in mammalian brain. Proceedings of the National Academy of Sciences of the United States of America. 2009; 106:2864-2867.

17. Kang TH, Sancar A. Circadian regulation of DNA excision repair: implications for chrono-chemotherapy. Cell cycle. 2009; 8:1665-1667.

18. Lee TH, Park JM, Leem SH, Kang TH. Coordinated regulation of XPA stability by ATR and HERC2 during nucleotide excision repair. Oncogene. 2014; 33:19-25.

19. Kang TH, Lindsey-Boltz LA, Reardon JT, Sancar A. Circadian control of XPA and excision repair of cisplatin-DNA damage by cryptochrome and HERC2 ubiquitin ligase. Proceedings of the National Academy of Sciences of the United States of America. 2010; 107:4890-4895.

20. Fan W, Luo J. SIRT1 regulates UV-induced DNA repair through deacetylating XPA. Molecular cell. 2010; 39:247-258.

21. Fang EF, Scheibye-Knudsen M, Brace LE, Kassahun H, SenGupta T, Nilsen H, Mitchell JR, Croteau DL, Bohr VA. Defective mitophagy in XPA via PARP-1 hyperactivation and NAD(+)/SIRT1 reduction. Cell. 2014; 157:882-896.

22. Dzagnidze A, Katsarava Z, Makhalova J, Liedert B, Yoon MS, Kaube H, Limmroth V, Thomale J. Repair capacity for platinum-DNA adducts determines the severity of cisplatin-induced peripheral neuropathy. The Journal of neuroscience : the official journal of the Society for Neuroscience. 2007; 27:9451-9457.

23. Gertz M, Fischer F, Nguyen GT, Lakshminarasimhan M, Schutkowski M, Weyand M, Steegborn C. Ex-527 inhibits Sirtuins by exploiting their unique NAD+-dependent deacetylation mechanism. Proceedings of the National Academy of Sciences of the United States of America. 2013; 110:E2772-2781.

24. Rebucci M, Michiels C. Molecular aspects of cancer cell resistance to chemotherapy. Biochemical pharmacology. 2013; 85:1219-1226.

25. Cheung-Ong K, Giaever G, Nislow C. DNA-damaging agents in cancer chemotherapy: serendipity and chemical biology. Chemistry \& biology. 2013; 20:648-659. 
26. Cramers P, Filon AR, Pines A, Kleinjans JC, Mullenders LH, van Zeeland AA. Enhanced nucleotide excision repair in human fibroblasts pre-exposed to ionizing radiation. Photochemistry and photobiology. 2012; 88:147-153.

27. Lin Z, Yang H, Kong Q, Li J, Lee SM, Gao B, Dong H, Wei J, Song J, Zhang DD, Fang D. USP22 antagonizes p53 transcriptional activation by deubiquitinating Sirt1 to suppress cell apoptosis and is required for mouse embryonic development. Molecular cell. 2012; 46:484-494.

28. Brunet A, Sweeney LB, Sturgill JF, Chua KF, Greer PL, Lin Y, Tran H, Ross SE, Mostoslavsky R, Cohen HY, Hu LS, Cheng HL, Jedrychowski MP, Gygi SP, Sinclair DA, Alt FW, et al. Stress-dependent regulation of FOXO transcription factors by the SIRT1 deacetylase. Science. 2004; 303:2011-2015.

29. Zhao W, Kruse JP, Tang Y, Jung SY, Qin J, Gu W. Negative regulation of the deacetylase SIRT1 by DBC1. Nature. 2008; 451:587-590.

30. Reardon JT, Sancar A. Purification and characterization of Escherichia coli and human nucleotide excision repair enzyme systems. Methods in enzymology. 2006; 408:189-213.

31. Joh HM, Choi JY, Kim SJ, Chung TH, Kang TH. Effect of additive oxygen gas on cellular response of lung cancer cells induced by atmospheric pressure helium plasma jet. Scientific reports. 2014; 4:6638. 